\title{
Comparison of outcomes in DeBakey type I versus DeBakey type II aortic dissection: a 17-year single center experience
}

\author{
Mona Salehi Ravesh ${ }^{1} \wedge$, Mohamed Salem ${ }^{2}$, Georg Lutter ${ }^{2}$, Christine Friedrich $^{2}$, Veronika Walter $^{2}$, \\ Thomas Puehler ${ }^{2}$, Jochen Cremer ${ }^{2}$, Assad Haneya ${ }^{2}$ \\ ${ }^{1}$ Department of Radiology and Neuroradiology, University Hospital Schleswig-Holstein Campus, Kiel, Germany; ${ }^{2}$ Department of Cardiovascular \\ Surgery, University Hospital Schleswig-Holstein Campus, Kiel, Germany \\ Contributions: (I) Conception and design: M Salem, G Lutter, V Walter, T Puehler, J Cremer, A Haneya; (II) Administrative support: J Cremer, A \\ Haneya; (III) Provision of study materials or patients: M Salem, G Lutter, V Walter, T Puehler, J Cremer, A Haneya; (IV) Collection and assembly of \\ data: A Haneya, C Friedrich; (V) Data analysis and interpretation: A Haneya, C Friedrich, M Salehi Ravesh; (VI) Manuscript writing: All authors; (VII) \\ Final approval of manuscript: All authors. \\ Correspondence to: Mona Salehi Ravesh, PhD. Department for Radiology and Neuroradiology, University Hospital Schleswig-Holstein, Arnold- \\ Heller-Street 3, Building D, 24105 Kiel, Germany. Email: Mona.Salehiravesh@uksh.de.
}

Background: It is controversial if the type or the size of aortic dissection is associated with the mortality in patients with acute aortic dissection (AAD) type I or type II according to DeBakey. Due to the pronounced aortic pathology in DeBakey type I compared to DeBakey type II, it is to be expected, that the DeBakey type I is associated with a significant higher morbidity and mortality. But we hypothesize that the current advances in surgical techniques, circulatory management, and postoperative care improve the clinical outcome of patients with DeBakey type I and II. The purpose of this study was to evaluate retrospectively the effect of these parameters on surgical outcome in patients with DeBakey type I and type II in a large cohort study.

Methods: From 2001 to 2019, 395 consecutive patients (34.2\% female) underwent surgical aortic repair at our institution. Patients were retrospectively classified into 2 groups: patients with type 1 dissection (group 1: $\mathrm{n}=309$, median age of 62.0 years) and patients with type 2 dissection (group 2: $\mathrm{n}=86,67.5$ years). Survival was estimated by Kaplan-Meier estimator. Risk factors were analyzed by logistic regression analysis.

Results: The patients in group 1 suffered significantly more often from coronary heart disease [43 (13.9\%) vs. 20 (23.3\%), $\mathrm{P}=0.036$ ]. Otherwise, there were no significant differences between both groups concerning preoperative risk factors. The median surgical duration ( 279 vs. $263 \mathrm{~min}, \mathrm{P}=0.026)$ and the circulatory arrest time (35 vs. $27 \mathrm{~min}, \mathrm{P}<0.001$ ) in group 1 were significantly higher. In a significantly higher number of patients in group 1 , the aortic arch was completely replaced $(18.4 \%$ vs. $1.2 \%, \mathrm{P}<0.001)$ and a simultaneous coronary artery bypass grafting [18 (5.8\%) vs. 11 (12.8\%), $\mathrm{P}=0.028]$ was performed. The rate of re-thoracotomy [62 (20.1\%) vs. 9 (10.5\%), $\mathrm{P}=0.040$ ], of postoperative delirium [66 (21.4\%) vs. $9(10.6 \%), \mathrm{P}=0.024]$, and of tracheotomy [85 (27.5\%) vs. $14(16.3 \%), \mathrm{P}=0.034$ ] were significantly higher in group 1 . Thirty-day mortality was $15.7 \%$ and did not differ significantly between both groups $(\mathrm{P}=0.867)$, as well as the long-term survival rates $(\mathrm{P}=0.956)$.

Conclusions: Due to the pronounced aortic pathology in type I compared to type II, it is to be expected, that the type $\mathrm{I}$ is associated with a significant higher morbidity and mortality. DeBakey type I was an independent predictor for 30-day mortality in our study, however, based on our 17-year single center experience there was no difference between the long-term survival in both groups.

Keywords: Aortic dissection; DeBakey type 1 and type 2; long-term surgical outcome; single center

Submitted May 09, 2021. Accepted for publication Sep 03, 2021.

doi: $10.21037 /$ jtd-21-809

View this article at: https://dx.doi.org/10.21037/jtd-21-809

^ ORCID: 0000-0003-2636-7508. 


\section{Introduction}

Acute aortic dissection (AAD) is a life-threatening medical emergency. It is associated with high rates of morbidity and mortality (1). The aortic dissections into three types (type I, II, and III) according to the DeBakey classification based upon the of the dissection (2). Type I dissections originate in the ascending aorta and propagate distally to the descending aorta for a variable distance. Type II dissections involve the ascending aorta only and type III dissections begin in the descending aorta, usually at or just distal to the left subclavian artery, and propagate above or below the diaphragm.

It is controversial if the type or the size of aortic dissection is associated with the mortality in patients with aortic dissection type I or type II (3-6). We hypothesize that the current advances in surgical techniques, circulatory management, and postoperative care improve the clinical outcome of patients with DeBakey type I and II The purpose of this study was therefore to evaluate retrospectively the effect of these parameters on the outcome in patients with aortic dissection type I and type II in a large cohort study. We present the following article in accordance with the STROBE reporting checklist (available at https://dx.doi.org/10.21037/jtd-21-809).

\section{Methods}

\section{Patients and study design}

We analyzed all patients with AAD enrolled in our Registry of AAD from January 2001 to May 2019. AAD is defined as the occurrence of a dissection involving the ascending aorta within 48 hours from the first onset of symptoms. In total, retrospectively 395 consecutive patients, who underwent replacement of aorta using moderate hypothermic circulatory arrest (MHCA) at $18-22{ }^{\circ} \mathrm{C}$, were analyzed. Patients were divided into two groups: group 1 (DeBakey type I) and group 2 (DeBakey type II). Data were supplied from the institution's database and medical records. Several independent surgeons were involved in the treatment of patients with $\mathrm{AAD}$, however, only senior surgeons with a longstanding surgical experience carry out those types of surgeries. Moreover, all surgeons followed the standard technique of our centre.

The diagnosis was confirmed preoperatively by a contrast enhanced computed tomography (CT) to detect the exact location and extension of the dissection membrane. Patients were investigated for neurological symptoms and questioned at admission for any history of neurological events. Postoperative neurological complications were consulted directly by a neurologist and categorized according to neurological assessment, followed by head and neck CT as well as, in many cases, CT angiography for the carotid arteries to estimate the extent of stroke and brain ischemia. Acute myocardial infarction (AMI) was detected through ECG changes and specific laboratory parameters of heart infarction. Patients with a residual dissection or organ malperfusion were treated additionally interventionally using intraoperatively thoracic endovascular aortic repair (TEVAR/EVAR).

The primary endpoints were 30-day mortality and postoperative neurological events [transient ischemic attack (TIA) and stroke]. Secondary endpoints were pre- and intraoperative variables, as well as the postoperative courses such as blood loss and transfusion of blood products.

The study was conducted in accordance with the Declaration of Helsinki (as revised in 2013). The study was approved by the Ethics Board of University of Kiel/ Germany (No. D417/17) and individual consent for this retrospective analysis was waived.

\section{Operative technique}

The cardiopulmonary bypass $(\mathrm{CPB})$ was performed with MHCA with a nasopharyngeal temperature between $18-22^{\circ} \mathrm{C}$. Arterial cannulation was performed either through cannulation of the distal ascending aorta or the femoral artery. Since 2010, the cannulation of the left ventricle transatrial via the right upper pulmonary was established as the standard (7). Venous drainage was performed either through cannulation of the femoral vein or the right atrium with common two-stage venous cannula. For myocardial protection a retrograde injection of cold blood cardioplegic solution was used. A bilateral antegrade cerebral perfusion with oxygenated cold blood $\left(18^{\circ} \mathrm{C}\right)$ was introduced through a balloon catheter inserted in arch vessels with a pressure control of 50-60 mmHg.

\section{Statistical analysis}

The statistical program SPSS 24.0 (SPSS, Chicago, IL, USA) was used for statistical analysis and calculation of the characteristic data. The frequency distribution of the sample data was examined for deviations from the normal distribution using the Kolmogorov-Smirnov-test. The mean \pm standard deviation was given for normally distributed, 
continuous variables. The median and the associated quartiles were given for values that were not normally distributed. Categorical variables were given using the number of affected patients (n) and a percentage (\%). The Chi-square test and, if necessary, the exact Fisher test were used to compare the two groups examined. Survival was calculated on rightcensored data by Kaplan-Meier analyses and was compared by $\log$ rank test. Follow-up completeness was $92.2 \%$ and $96.5 \%$ for DeBakey 1 and DeBakey 2, respectively and follow-up duration was $10.97(8.67-13.26)$ years and $9.06(6.81-11.31)$ years, respectively.

Variables associated with 30-day mortality in univariate statistics, age, gender, DeBakey type, preoperative arterial hypertension, atrial fibrillation and coronary heart disease, and intraoperatively TEVAR and number of red blood cell concentrates were selected for multiple logistic regression analysis to determine their relative impact (adjusted odds ratio, OR) on 30-day mortality with a goodness of fit, described by Cox-Snell-R-Squared, of 0.153 . The predictive value of the logistic regression model was estimated by the Hosmer-Lemeshow $\chi^{2}$ test $(\mathrm{P}=0.737)$. All $\mathrm{P}$ values $<0.05$ were rated as a significant difference between the two groups.

\section{Results}

\section{Demographics and clinical characteristics of the study population}

Relevant demographics and preoperative data of patients are given in Table 1. There were no significant differences between the patients in both groups concerning the age and gender. Group 2 included significantly more patients with pulmonary hypertension [3 (1\%) vs. 4 (4.7\%), $\mathrm{P}=0.042]$. The patients in group 1 had a significantly higher left ventricular ejection fraction $[60.5 \%(55 ; 70)$ vs. $60 \%$ (48; 69), $\mathrm{P}=0.013]$. A significantly lower percentage of patients in group 1 suffered from coronary heart disease [43 (13.9\%) vs. 20 (23.3\%), $\mathrm{P}=0.036$ ].

\section{Intraoperative data}

The median surgery duration of patients in group 1 was significantly higher than that of patients in group 2 [279 (230; 345) vs. $263(210 ; 311)$ min, $\mathrm{P}=0.026]$. The duration of circulatory arrest in patients of group 1 was significantly higher $[35(27 ; 57)$ vs. $27(20 ; 35) \mathrm{min}, \mathrm{P}<0.001]$, as well as cross-clamp time. The aortic arch was completely replaced in a significantly higher number of patients in group 1 [57 (18.4\%) vs. 1 (1.2\%), $\mathrm{P}<0.001]$. A significantly higher number of patients in group 1 additionally had a simultaneous coronary artery bypass grafting $[18(5.8 \%) v s$. $11(12.8 \%), \mathrm{P}=0.028]$ and more patients in group 1 required fresh frozen plasma [145 (47.1\%) vs. 29 (33.7\%), $\mathrm{P}=0.027$ ]. Otherwise no significant differences were noted between the two groups with regard to intraoperative data (Table 2).

\section{Postoperative data and outcomes}

The postoperative data and surgical outcomes of patients in both groups are presented in Table 3. A significantly higher number of patients in group 1 underwent a re-thoracotomy [62 (20.1\%) vs. 9 (10.5\%), $\mathrm{P}=0.040$ ] and received platelets [149 (49.5\%) vs. 30 (35.7\%), $\mathrm{P}=0.025]$, but the total number of received platelets was significantly higher in patients of group 2 [0 (0-20) vs. $1(0-11)$ unit, $\mathrm{P}=0.028]$. A tracheotomy was more often performed in patients of group 1 [85 (27.5\%) vs. 14 (16.3\%), $\mathrm{P}=0.034$ ]. A postoperative delirium was significantly more frequent in patients of group 1 [66 (21.4\%) vs. 9 (10.6\%), $\mathrm{P}=0.024]$. The patients in group 1 stayed for a significantly longer time in the ICU $[6(3 ; 13)$ vs. $4(2 ; 8)$ day, $\mathrm{P}=0.018]$. The postoperative in hospital stay was significantly longer in group 1 [11 $(7 ; 20)$ vs. $9(4 ; 15)$ day, $\mathrm{P}=0.034]$. There were no differences between both patient groups concerning neurological events.

Based on the logistic regression analysis results DeBakey type $I$ is a significant independent risk factor for 30-day mortality in patients operated on for AAD. Further significant risk factors in our study were male gender, arterial hypertension, atrial fibrillation, coronary heart disease, TEVAR and intraoperatively administered number of red blood cell concentrates (Table 4).

Short- and long-term survival were in the same range in both groups. The 1-year (76\% vs. 79\%), 3-year (74\% vs. $74 \%$ ), 5 -year (69\% vs. $73 \%$ ), 7 -year (61\% vs. $66 \%$ ), and 10 -year $(51 \%$ vs. $45 \%)$ survival rates were in the same range in both groups $(\mathrm{P}=0.956)$ (Figure 1).

\section{Discussion}

In our study, the short- and long-term surgical outcome of 395 consecutive patients with two different kinds of aortic dissections was investigated. Three hundred and nine patients (78.2\% of the cohort) were identified as DeBakey type 1 and 86 patients (21.8\% of the cohort) as DeBakey 
Table 1 Demographic and clinical characteristics of the study population

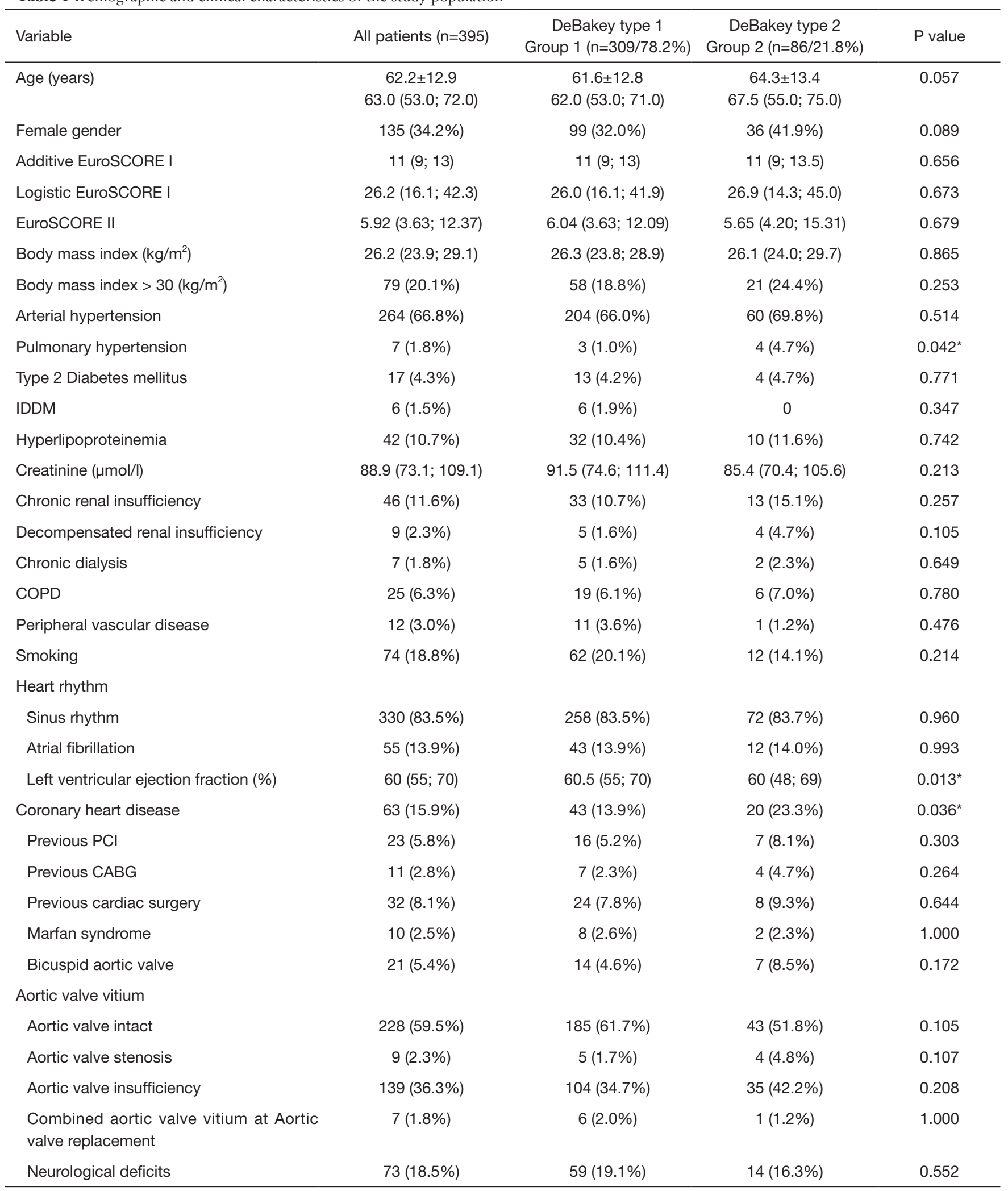

Table 1 (continued) 
Table 1 (continued)

\begin{tabular}{|c|c|c|c|c|}
\hline Variable & All patients $(n=395)$ & $\begin{array}{c}\text { DeBakey type } 1 \\
\text { Group } 1(n=309 / 78.2 \%)\end{array}$ & $\begin{array}{c}\text { DeBakey type } 2 \\
\text { Group } 2(n=86 / 21.8 \%)\end{array}$ & $P$ value \\
\hline \multicolumn{5}{|l|}{ Clinical presentation } \\
\hline Cardiogenic shock & $29(7.4 \%)$ & $22(7.1 \%)$ & $7(8.2 \%)$ & 0.727 \\
\hline CRP ( $\leq 48 \mathrm{~h})$ & $29(7.3 \%)$ & $21(6.8 \%)$ & $8(9.3 \%)$ & 0.431 \\
\hline Intubated at admission & $40(10.2 \%)$ & $31(10.0 \%)$ & $9(10.6 \%)$ & 0.881 \\
\hline Pericardial tamponade & $64(16.2 \%)$ & $45(14.6 \%)$ & $19(22.1 \%)$ & 0.096 \\
\hline
\end{tabular}

*, significant $\mathrm{P}$ value. IDDM, Insulin-dependent diabetes mellitus; COPD, chronic obstructive pulmonary disease; PCI, percutaneous coronary intervention; CABG, coronary artery bypass grafting; CRP, c-reactive protein.

type 2. Both groups were compared concerning their demographic, pre-, intra- and postoperative data. There were no differences between both groups concerning their demographic data. However, there were significant differences between both groups concerning some of the co-morbidities, intra-, and postoperative data. Although the patients with DeBakey type 1 have more complex aortic dissection and significant longer surgical duration, ICU time and postoperative in-hospital days, there is no significant difference between these patients and those with DeBakey type 2 concerning the 7-day and 30-day mortality and also long-term outcome.

In the literature, there are only few studies about the investigation of surgical outcomes of patients with DeBakey type I or II (3-6).

Glower et al. (5) investigated the management and long-term outcomes of 163 patients with three types of aortic dissection in a single-center study in 1991. In total, 66 patients in both subgroups I and II were 10 years younger than our patients. In contrast to our study Glower et al. observed differences between the aortic dissection subgroups concerning the 30-day mortality and 5-year survival. The intraoperative mortality rate was lower for type I patients than that for type II patients (11\% vs. 14\%). The 30 -day mortality rates were $26 \%$ for type I patients and $14 \%$ for type II patients and 5 -year survival rates were $56 \% \pm 9 \%$ vs. $87 \% \pm 8 \%$. Glower et al. reported that 30-day mortality rates in patients with acute types I or II dissection appeared to be dependent on the experience of the operating surgeon.

Based on the available publications in the literature, Trimarchi et al. (4) reported in 2004 that surgical mortality for acute DeBakey type I and II aortic dissection in different studies from single centers or surgeons varies from $7 \%$ to $30 \%$. For the identification of a preoperative risk stratification scheme and a real average surgical mortality, Trimarchi et al. collected patients from 18 referral centers worldwide on behalf of the international registry of AAD investigators. They performed a comprehensive analysis of 290 clinical variables and their relationship to surgical outcomes in the study patients. According to risk profile of patients, they were categorized in unstable and stable condition group (I and II). The overall in-hospital mortality was $25.1 \%$. Mortality in group I was significantly higher compared to that in group II (31.4\% vs. $16.7 \%)$. Independent preoperative predictors of operative mortality were history of aortic valve replacement, migrating chest pain, hypotension as sign of acute type I/II aortic dissection, shock or tamponade, preoperative cardiac tamponade and preoperative limb ischemia. Based on this analysis, Trimarchi et al. confirmed that patient selection plays an important role in determining surgical outcomes in patients with acute type I/II aortic dissection. Knowledge of significant risk factors for operative mortality can result in a better management and a more defined risk assessment in these patients.

In contrast to the study of Trimarchi et al. the overall inhospital mortality of our patients was lower than $15 \%$, the 7 day-mortality in DeBakey group I was not significantly lower than of the group II (9.4\% vs. 11.6\%), whereas the 30 day-mortality of both groups was in the same range.

Easo et al. (6) analyzed in 2013 the influence of operative strategy for the aortic arch on surgical outcomes of patients in DeBakey type I aortic dissection. They included 658 
Table 2 Operative data

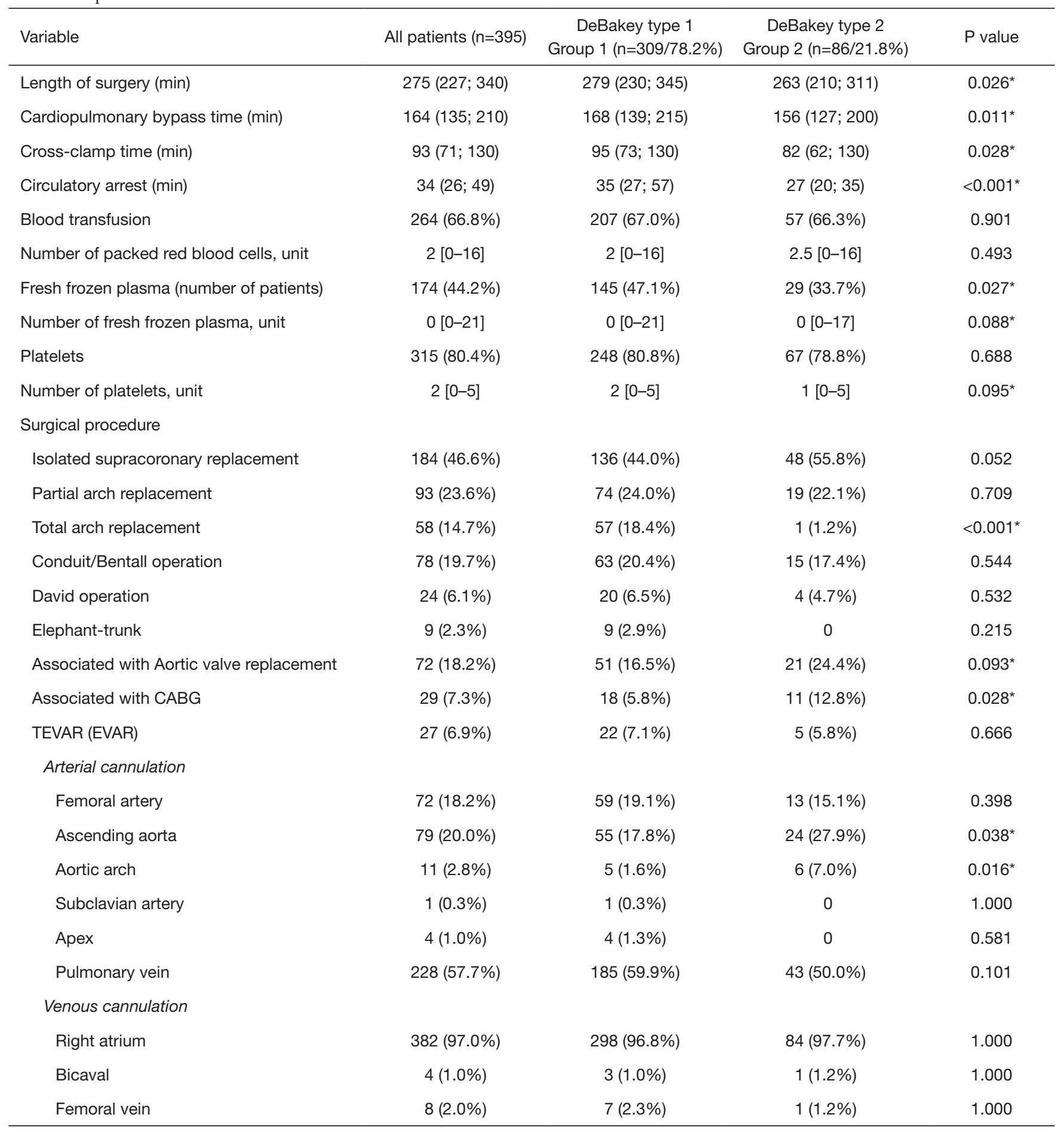

*, significant $\mathrm{P}$ value. CABG, coronary artery bypass grafting; TEVAR, thoracic endovascular aortic repair; EVAR, endovascular aortic repair. 
Table 3 Postoperative data and outcomes

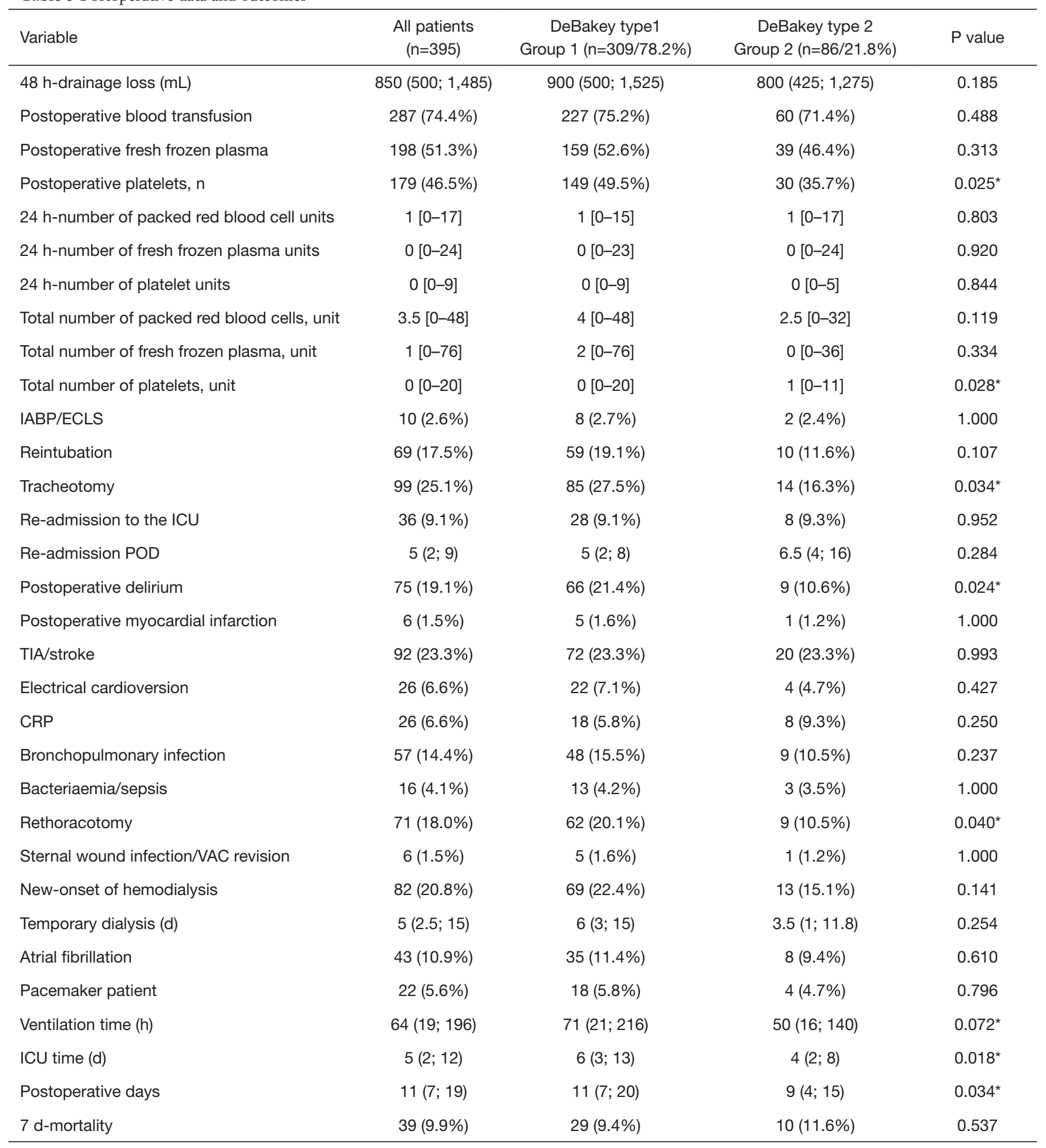

Table 3 (continued) 
Table 3 (continued)

\begin{tabular}{|c|c|c|c|c|}
\hline Variable & $\begin{array}{l}\text { All patients } \\
\quad(\mathrm{n}=395)\end{array}$ & $\begin{array}{c}\text { DeBakey type1 } \\
\text { Group } 1(n=309 / 78.2 \%)\end{array}$ & $\begin{array}{c}\text { DeBakey type } 2 \\
\text { Group } 2(n=86 / 21.8 \%)\end{array}$ & $P$ value \\
\hline 30 d-Mortality & $62(15.7 \%)$ & 49 (15.9\%) & $13(15.1 \%)$ & 0.898 \\
\hline Hospital Mortality & $58(14.7 \%)$ & $45(14.6 \%)$ & $13(15.1 \%)$ & 0.898 \\
\hline Cardiac death & $32(55.2 \%)$ & 25 (55.6\%) & 7 (53.8\%) & 0.884 \\
\hline MOF & $18(31.0 \%)$ & $14(31.1 \%)$ & $4(30.8 \%)$ & 0.884 \\
\hline
\end{tabular}

*, significant $\mathrm{P}$ value. IABP, intra-aortic balloon bump; ECLS, extracorporeal life support; ICU, intensive care unit; POD, postoperative day;

TIA, transient ischemic attack; CRP, C-reactive protein; VAC, vacuum-assisted closure; MOF, multiple organ failure.

Table 4 Logistic regression analysis on pre- and intraoperative factors for combined endpoint (tracheotomy, rethoracotomy, delir, ICU >5 days) in AADA patients

\begin{tabular}{lccc}
\hline Predictors & Odd ratio & $95 \% \mathrm{Cl}$ & $\mathrm{P}$ value \\
\hline DeBekey type 1 & 2.230 & $1.323-3.757$ & $0.003^{*}$ \\
Male gender & 1.703 & $1.075-2.699$ & $0.023^{*}$ \\
Arterial hypertension & 1.772 & $1.126-2.789$ & $0.013^{*}$ \\
Atrial fibrillation & 2.094 & $1.055-4.156$ & $0.035^{*}$ \\
Coronary heart disease & 2.044 & $1.075-3.886$ & $0.029^{*}$ \\
TEVAR (EVAR) & 3.373 & $1.114-10.212$ & $0.031^{*}$ \\
Number of RBC & 1.080 & $1.010-1.155$ & $0.025^{*}$ \\
\hline
\end{tabular}

*, significant $P$ value. ICU, intensive care unit; AADA, acute aortic dissection type A; TEVAR, thoracic endovascular aortic repair; EVAR, endovascular aortic repair; RBC, red blood cell.

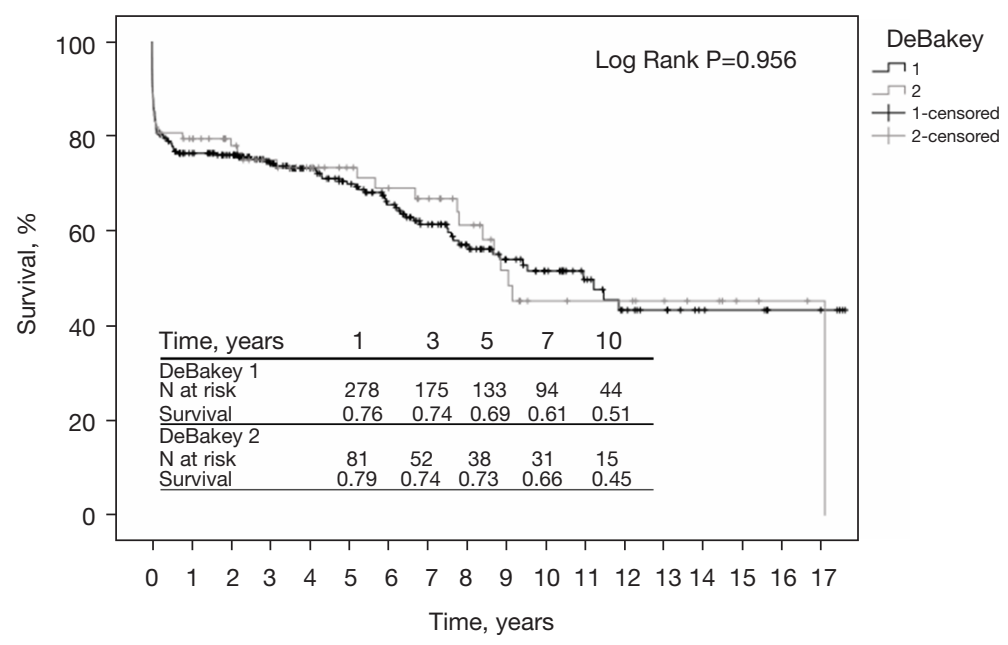

Figure 1 The estimated survival curves by Kaplan-Meier method. 
patients from the German registry for AAD type I. The patients were divided into two groups. Group A had conventional treatment with replacement of the ascending aorta and hemiarch replacement/open distal anastomosis. Group B had extensive surgery with complete arch replacement, possibly in combination with an elephant trunk/frozen elephant trunk treatment of the descending aorta. In accordance to our study, the mean operative time and circulatory arrest time were significantly shorter in group A than in group B. Postoperative outcomes showed a higher rate of rethoracotomy, bleeding, staying in ICU, and in-hospital day for group B. Overall mortality was 133 patients $(20.2 \%)$, with a tendency for lower rate of mortality in Group A (18.7\%) than in Group B (25.7\%). Based on these results Easo et al. reported that more extensive treatment with total arch replacement and possibly adjunct therapy of the descending aorta can be performed in an acceptable operative risk comparable to the standard treatment with replacement of the ascending aorta. Immediate postoperative complications are higher; however, 30-day mortality show no significant difference.

Both overall in-hospital mortality of our patients and 7 day-mortality as well as 30 day-mortality in both groups were lower than corresponding data from the study of Easo et al. In agreement with this study, there were no differences between both DeBakey group in our study regarding to the 30 day-mortality $(\mathrm{P}=0.898)$.

In 2018, a multicentric and international study from Kohl et al. (3) was published which is comparable to our work concerning the comparison of outcomes of a cohort of patients with DeBakey type II in contrast to DeBakey type I. This study included in total, 1,691 patients with DeBakey type I und 181 patients with DeBakey type II from 37 referral centers in 11 countries. It considered a medium survival time of 5 years for the investigation of outcomes. In contrast, we analyzed the patient's data over 17 years from our institutional registry of AAD. There were no differences between our study and this multicentric study concerning the age and percentage of female gender of all patients in the cohort. But the patients with DeBakey type II in this multicentric study were significantly younger, had a lower BMI, and included more female patients than those with DeBakey type I. Contrary to this study, there were no differences between our DeBakey groups concerning these demographic data. Our patients with DeBakey type II suffered significantly more often from pulmonary hypertension than those with DeBakey type I. In contrast, there is no difference between both DeBakey groups of the multicentric study concerning the history of pulmonary hypertension. Contrary to our study, in the multicentric study, not all of patients, but only $87.2 \%$ of patients with DeBakey I and 83.4\% with DeBakey II treated surgically. In agreement with our study, in the multicentric study the complete aortic arch was replaced in a significant higher number of patients with DeBakey I compared to DeBakey II. In the multicentric study in accordance with our study, there was no difference between both DeBakey types concerning the hospital mortality and 5-year survival by surgical treatment. 30-day mortality was not given in this study.

Due to the pronounced aortic pathology in DeBakey type I compared to DeBakey type II, it is to be expected, that the DeBakey type I is associated with a significant higher morbidity and mortality $(8,9)$. But the current advances in surgical techniques, circulatory management, and postoperative care improve the clinical outcome of patients with DeBakey type I and II (10).

\section{Conclusions}

Based on our 17-year single center experience, we compared the clinical outcomes in DeBakey type I $v s$. DeBakey type II aortic dissection. Due to the distinct aortic pathology in DeBakey type I compared to DeBakey type II, it is to be expected, that the DeBakey type I results in a significant higher morbidity and mortality. While there was no difference in unadjusted short- and long-term survival between both groups, DeBakey type I revealed as independent predictor for 30-day mortality. Further prospective, multicentric, and randomized clinical studies with a larger group of patients are required to investigate in detail the effect of different surgical methods on long-term clinical outcome in patients with DeBakey type I and II.

\section{Limitations}

The main limitation of this study is the retrospective design of this study and the inhomogeneity of the two study groups. Multivariable logistic regression analysis was performed based on this large sample size to adjust for known confounders, however, there still remains a risk of unknown or not surveyed confounders.

\section{Acknowledgments}

Funding: This study was supported by The German Center of Cardiovascular Research (DZHK). 


\section{Footnote}

Reporting Checklist: The authors have completed the STROBE reporting checklist. Available at https://dx.doi. org/10.21037/jtd-21-809

Data Sharing Statement: Available at https://dx.doi. org/10.21037/jtd-21-809

Peer Review File: Available at https://dx.doi.org/10.21037/ jtd-21-809

Conflicts of Interest: All authors have completed the ICMJE uniform disclosure form (available at https://dx.doi. org/10.21037/jtd-21-809). The authors have no conflicts of interest to declare.

Ethical Statement: The authors are accountable for all aspects of the work in ensuring that questions related to the accuracy or integrity of any part of the work are appropriately investigated and resolved. The study was conducted in accordance with the Declaration of Helsinki (as revised in 2013). The study was approved by the Ethics Board of University of Kiel/Germany (No. D417/17) and individual consent for this retrospective analysis was waived.

Open Access Statement: This is an Open Access article distributed in accordance with the Creative Commons Attribution-NonCommercial-NoDerivs 4.0 International License (CC BY-NC-ND 4.0), which permits the noncommercial replication and distribution of the article with the strict proviso that no changes or edits are made and the original work is properly cited (including links to both the formal publication through the relevant DOI and the license). See: https://creativecommons.org/licenses/by-nc-nd/4.0/.

\section{References}

1. Hagan PG, Nienaber CA, Isselbacher EM, et al. The

Cite this article as: Salehi Ravesh M, Salem M, Lutter G, Friedrich C, Walter V, Puehler T, Cremer J, Haneya A. Comparison of outcomes in DeBakey type I versus DeBakey type II aortic dissection: a 17-year single center experience. J Thorac Dis 2021;13(12):6769-6778. doi: 10.21037/jtd-21-809
International Registry of Acute Aortic Dissection (IRAD): new insights into an old disease. JAMA 2000;283:897-903.

2. Debakey ME, Henly WS, Cooley DA, et al. Surgical management of dissecting aneurysms of the aorta. J Thorac Cardiovasc Surg 1965;49:130-49.

3. Kohl LP, Isselbacher EM, Majahalme N, et al. Comparison of Outcomes in DeBakey Type AI Versus AII Aortic Dissection. Am J Cardiol 2018;122:689-95.

4. Trimarchi S, Nienaber CA, Rampoldi V, et al. Contemporary results of surgery in acute type A aortic dissection: The International Registry of Acute Aortic Dissection experience. J Thorac Cardiovasc Surg 2005;129:112-22.

5. Glower DD, Speier RH, White WD, et al. Management and long-term outcome of aortic dissection. Ann Surg 1991;214:31-41.

6. Easo J, Weigang E, Hölzl PP, et al. Influence of operative strategy for the aortic arch in DeBakey type I aortic dissection - analysis of the German Registry for Acute Aortic Dissection type A (GERAADA). Ann Cardiothorac Surg 2013;2:175-80.

7. Schoeneich F, Rahimi-Barfeh A, Grothusen C, et al. Transatrial left-ventricular cannulation in acute aortic dissection type A: a novel cannulation technique. Eur J Cardiothorac Surg 2015;48:e51-2.

8. Geirsson A, Bavaria JE, Swarr D, et al. Fate of the residual distal and proximal aorta after acute type a dissection repair using a contemporary surgical reconstruction algorithm. Ann Thorac Surg 2007;84:1955-64; discussion 1955-64.

9. Kazui T, Washiyama N, Muhammad BA, et al. Total arch replacement using aortic arch branched grafts with the aid of antegrade selective cerebral perfusion. Ann Thorac Surg 2000;70:3-8; discussion 8-9.

10. Frankel WC, Green SY, Orozco-Sevilla V, et al. Contemporary Surgical Strategies for Acute Type A Aortic Dissection. Semin Thorac Cardiovasc Surg 2020;32:617-29. 